\title{
APLICACIONES INNOVADORAS DE LA MADERA EN LA CONSTRUCCIÓN
}

\section{(WOOD INNOVATIVE APPLICATIONS IN CONSTRUCTION)}

\author{
Justo Garcia Navarro, Dr. Arquitecto \\ Profesor Titular de la Universidad Politécnica de Madrid. Asesor de la Fundación Cultural COAM \\ Ignacio Martínez Elcoro, Ingeniero de Montes \\ Consultor de APA-The Engineered Wood Association
}

ESPAÑA

Fecha de recepción: 25-X-96

\section{RESTIMEN}

La utilización de la madera como material de construcción ha sufrido un profundo cambio en el último medio siglo. Las nuevas tecnologias y procesos industriales han provocado, junto a la optimización de las caracteristicas intrinsecas del material, un mejor aprovechamiento y utilización de la madera, tanto para usos estructurales como para usos no resistentes.

Este articulo, que recoge algunos de los temas desarrollados en las distintas Jornadas y Cursos sobre Construcción en Madera, organizados por la Fundación Cultural COAM durante los últimos dos años, pretende hacer un repaso no exhaustivo a las diferentes aplicaciones del material, haciendo especial hincapié en los aspectos más innovadores que, por diversas circunstancias, no han sido, hasta la fecha, suficientemente utilizados en España.

\section{SUMMARY}

The use of wooden products in construction has experienced significant changes during the last decades. New technologies have allowed to produce better and more predictable products for structural applications while have improved transformation processes to make more efficient use of the available raw resource.

This paper describes current applications of new wooden materials uncommon in the Spanish construction. The Fundacion Cultural COAM has channeled this information to designers in a number of seminars and courses during the last two years with special attention to the innovative applications.

\section{Introducción}

La evolución de la construcción con madera camina hacia una sustitución de la madera maciza por materiales industrializados-madera industrializada-, limitando el uso de aquélla a aplicaciones de interior y decorativas. El empleo creciente de productos estructurales de madera viene dictado por numerosas circunstancias, entre las que destacan:
- La creciente escasez de madera de grandes dimensiones

- La demanda social hacia una mayor protección de los recursos naturales y el uso múltiple de los recursos forestales

- La mejora de las tecnologías de producción de productos encolados, asi como de las colas

- La necesidad de ahorro de materia prima

- La adaptación a las normativas de construcción 
Durante cientos de años, la madera ha sido el material de construcción natural para viviendas y todo tipo de estructuras en Europa. La revolución tecnológica e industrial del último siglo introdujo nuevos materiales estructurales, lo que significó un progresivo abandono de la madera. Aunque este proceso se mantiene, e incluso es previsible que se incremente, se vuelve otra vez a considerar el uso de la madera, aunque con un enfoque diferente.

De esta manera, la madera vuelve a ser un recurso interesante para arquitectos, ingenieros y constructores debido a la mejora de su tecnología. Los últimos desarrollos han aumentado la durabilidad de la madera, generado una amplia familia de nuevos productos que han rebasado las limitaciones de ésta en su forma original gracias a las colas, y han contribuido al diseño de unos medios de unión capaces de transmitir mayores cargas con una fiabilidad superior.

Al igual que en otros materiales de construcción, la madera, y en mayor medida sus derivados, están disponibles en una amplia variedad de calidades y tamaños normalizados. Los fabricantes de estos productos se han integrado en asociaciones para facilitar el proceso de normalización de calidades y tamaños, y asegurar que las normas y un nivel de calidad mínimo se mantengan. De la misma forma, también los procesos de tratamiento se han normalizado, y están sujetos a control de calidad.

No obstante, la producción industrial de elementos compuestos ha ido por delante del proceso normalizador. Ello ha requerido de los fabricantes la gestión y obtención de certificados de garantía que avalen sus productos en un ámbito nacional.

\section{Los tableros de madera}

Ha sido en el transcurso del siglo XX cuando se ha desarrollado toda la familia de tableros de madera merced a la mejora de la química de adhesivos.

El primero en aparecer fue el tablero contrachapado, que en su inicio sólo tuvo aplicaciones en carpintería. No fue hasta finales de los años 30, cuando se empezó a utilizar en construcción. Durante la misma década dio comienzo la producción industrial del tablero aglomerado, seguido por el tablero de fibras duro, en los años 40. Bajo un concepto parecido, pero con características bien diferenciadas, se incorpora el tablero de densidad media a la familia de paneles de madera, a comienzos de los 70 . Por la misma época, comienzan los ensayos del tablero de virutas, de disposición aleatoria -Waferboard-, o bien orientada (también conocido en el comercio y la literatura científica como OSB por sus siglas en inglés -Oriented Strand Board-). Finalmente, se ha añadidoel tablero de partículascemento, dotado de propiedades claramente diferenciadas del resto.
Todos ellos significaron en su momento una aportación novedosa para dar respuesta a las necesidades tecnológicas planteadas. En principio, cualquiera de ellos puede ser utilizado en diferentes aplicaciones de construcción, aunque algunos reúnen propiedades que los hacen especialmente indicados para usos estructurales. Los tableros tradicionalmente más usados en construcción son los contrachapados, aglomerados y, más recientemente, el OSB. El términoestructural implicaque su función principal es la de resistir las acciones de las fuerzas externas aplicadas sobre el panel para una aplicación concreta.

Todos los tableros estructurales de madera son materiales compuestos. Es decir, se trata de un compuesto de elementos lígneos tales como chapas, partículas, fibras o virutas, y un producto adhesivo. El contrachapado es un conjunto de chapas encoladas entre sí con orientación a 90 grados entre chapas sucesivas, siendo el más común de los llamados tableros estructurales. El aglomerado es una combinación de partículas y un adhesivo, y se utiliza profusamente en construcción. El OSB es un derivado en el que la geometría de la partícula, su orientación en el tablero y la disposición en capas, lo dotan de unas características similares en algunos aspectos al contrachapado y, por tanto, compite con éste en numerosas aplicaciones de construcción. Sin ser considerado propiamente como un tablero estructural, debido a su relativamente baja resistencia a flexión, el tablero de partículas-cemento tiene un excepcional comportamiento a la acción de la intemperie, así como frente al fuego.

La aplicación típica de los tableros estructurales de madera es la de forrar con un material fuerte y rígido una estructura relativamente flexible y esbelta (Fig. 1). Esta situación se da en los entramados ligeros de madera. Así, los tableros cosen los pies derechos de los entramados en paredes, cubierta y forjados, formando un todo rígido. Este forro queda oculto por el recubrimiento exterior o fachada visible. En ocasiones, se utiliza un tablero estructural

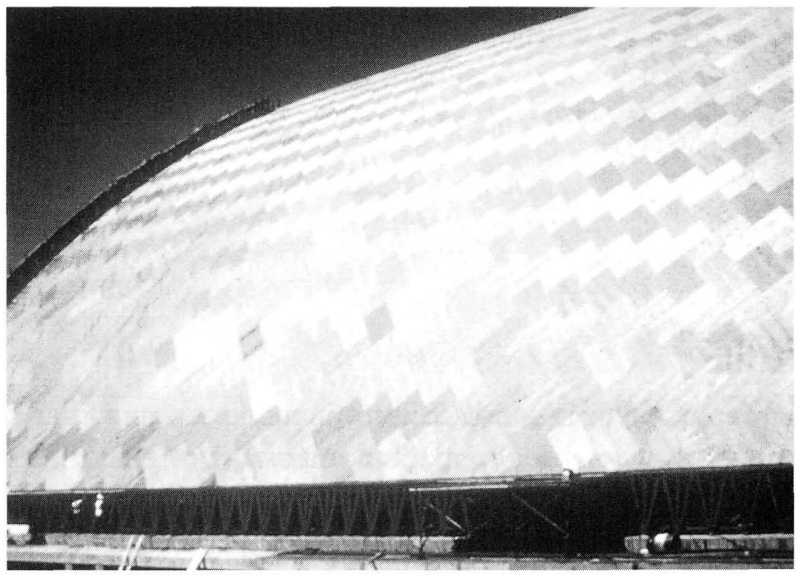

Fig. 1.- Revestimiento de la estructura. Kibbie Stadium, Universidad de Idaho, Moscow (EEUU). 


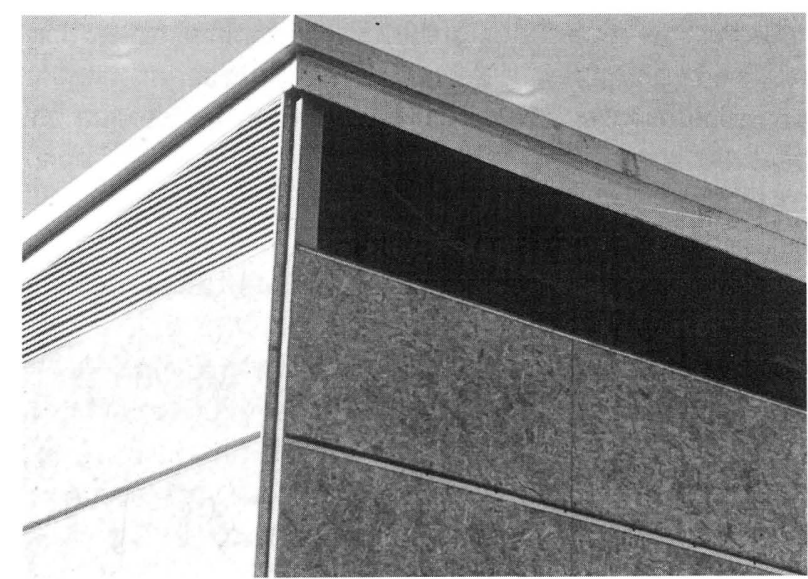

Fig. 2.- Revestimiento para exterior con tablero de OSB. Almacén editorial en Montgat (Barcelona).

específico de recubrimiento exterior que realiza la función estructural y la decorativa de acabado. En este caso, el tablero reune unas calidades superiores que lo cualifican para su plena exposición a la intemperie, además de un encolado fenólico, común por otra parte a todos los tableros estructurales (Fig. 2).

Adicionalmente se le puede dar un tratamiento decorativo superficial imitando un entablado, y normalmente se aumenta también su rugosidad para conseguir un aspecto de madera aserrada. En todo caso el tablero debe ser protegido con tintes más o menos opacos, en función del aspecto y frecuencia de mantenimiento que se deseen, o con pinturas opacas al agua, lo que le proporciona una protección más duradera. Aunque el tablero soporta perfectamente las inclemencias del tiempo sin que se le aplique ninguna protección, su color sin embargo torna irregularmente hacia los grises, proceso, por otra parte, idéntico al que se produce en la madera.

Con creciente importancia por sus posibilidades de prefabricación, el tablero contrachapado se usa en subsistemas estructurales, como vigas y columnas cajón o secciones enteras de cubierta -stressed skin panels- y otros conjuntos modulares. Es previsible que en un futuro también se utilicen para esta aplicación específica los aglomerados estructurales.

Debido a que históricamente el tablero contrachapado fue el primero en ser utilizado para la construcción, sus prestaciones se utilizan como referencia para evaluar las de otros tableros de madera en similares aplicaciones. Para ello hay tres áreas que se deben considerar. En primer lugar, la descripción detallada de los requisitos que debe cumplir en usoy las condiciones a que estará sometido. También se precisa una información detallada y fiable de las propiedades y comportamiento del material, como base de partida para un diseño riguroso. Finalmente, se debe valorar el tablero no sólo desde su valor estructural, sino en términos de economía, facilidad de instalación y mantenimiento, disponibilidad y consecuencias posibles en caso de rotura.

Examinando producto por producto sus propiedades estructurales, es difícil establecer una clara ventaja de unos sobre otros, pues depende del uso pretendido y las condiciones bajo las cuales será utilizado. Si se atiende, por ejemplo, a la resistencia a flexión de los distintos tableros, se observa que el DM alcanza los valores máximos. Sin embargo, la deformación ocasionada por carga permanente es superior al resto, además de la emisión de formaldehido, problema por lo demás que comparte con el aglomerado. El tablero de partículas-cemento tiene una resistencia a flexión relativamente baja pero la mantiene prácticamente inalterable con el tiempo. El OSB se sitúa en una posición intermedia respecto a esta propiedad. Aunque normalmente se fabrica con el mismo tipo de adhesivo que el tablero contrachapado de pino para uso exterior, sin embargo es dimensionalmente más inestable que aquél, sobre todo en el grueso, e incluso más que algunos tableros aglomerados en determinadas circunstancias. Esto se debe a que el OSB es un producto de una densidad notablemente superior a la de la madera con que se fabrica. El proceso de prensado induce tensiones en el interior del tablero que se disipan en cuantía variable cuando se expone a la humedad durante períodos suficientemente prolongados. El tablero contrachapado de pino dispone de una resistencia inicial a flexión que es la más alta de todos los tableros de madera, disminuyendo con el tiempo en mucha menor cuantía que en el resto, siendo su estabilidad dimensional aceptable. Sometido a procesos de envejecimiento, experimenta un apreciable agrietamiento superficial, particularmente en ensayos de ciclos de humedad-secado, que lo pueden contraindicar para determinadas aplicaciones.

Se puede concluir que los valores de ensayos de diferentes clases de tableros dependen del ensayo que se realiza, y que por tanto no hay una clase de tablero claramente superior a los demás en base a razones tecnológicas o económicas, ya que, de lo contrario, sólo se produciría ese tipo de tablero. La elección del tablero vendrá determinada por el uso a que se destina y otras consideraciones no relacionadas con sus características estructurales.

Como se ha indicado anteriormente, los tableros estructurales encuentran su campo de aplicación natural en la construcción con entramados ligeros de madera. Ello no obstante, también se pueden aplicar con ventaja en obra tradicional para aplicaciones en que su bajo peso y prestaciones así lo sugieren. Específicamente, la cubierta del edificio es una parte del mismo que debe ser considerada cuidadosamente, por el efecto que provocaría una acumulación excesiva de cargas -que se repercutiría sobre la estructura-, y por el aislamiento térmico del 
conjunto, principalmente. Con una mayor conciencia de la necesidad de mejorar la eficiencia energética en la edificación, la madera es una vez más una solución razonable y suficientemente probada. Con el empleo de los nuevos productos estructurales, se da respuesta a estas exigencias.

\section{La protección al fuego en los edificios con estructura de madera}

Se dan varias aproximaciones a este problema, con el objeto de proporcionar la necesaria seguridad frente al fuego de cualquier estructura. Se trata de una combinación de factores:

1 ) prevenir la posibilidad de fuego

2) controlar su crecimiento y expansión

3) proporcionar protección a las vidas y propiedades

Un diseño de protección contra fuego que minimice al máximo esos riesgos debe ser alcanzable con un costo reducido

El diseño de la estructura debe considerar un control en el desarrollo del fuego, de forma que se prevea la salida de los ocupantes o su protección en el interior de determinadas áreas, la constricción del siniestro en zonas de fuego cerradas y la inclusión de dispositivos para detección de humo y apagado del fuego en zonas críticas.

Existen igualmente tratamientos retardadores de la combustión a base de impregnación. El efecto destructor del fuego en este caso y la transmisión de calor se reducen por efecto del tratamiento, aunque lo más importante es disminuir la velocidad de expansión de la llama. Si la madera bajo el fuego ha absorbido suficiente cantidad de producto, tan pronto como cese su causa se apagará. Los tratamientos pueden ser en profundidad o superficiales. Estos últimos deben ser renovados periódicamente, aun en aplicaciones interiores. En caso de tratamientos en profundidad, se realizan de la misma manera que en los de protección frente a organismos patógenos. El inconveniente más importante de estos últimos, a largo plazo, es que reducen sensiblemente la resistencia de la madera y, en ocasiones, los productos químicos que intervienen provocan la corrosión de los conectores metálicos. Se ha comprobado una interacción perjudicial para la madera y, sobre todo, para el tablero contrachapado ignifugado en autoclave, cuando se aplica en tejados en condiciones de alta temperatura y humedad, por lo que es preciso asegurar la necesaria ventilación.

Con independencia de ello, existen técnicas de construcción en estructura ligera que, sin tratamientos específicos, proporcionan una resistencia al fuego, de duración ensayada en laboratorio, normalmente de una hora. Se utilizan tableros de yeso-cartón en todos los casos y diversos espesores de materiales aislantes no combustibles. En áreas no registrables, se deben prever barreras antifuego y movimientos convectivos del aire (corrientes de aire en zonas no accesibles). Normalmente se utilizan los mismos tableros y maderas que para el resto de la construcción.

\section{Los nuevos productos industrializados de mader:}

Laintroducción de componentes derivados de la madera en construcción como alternativa al hormigón, elementos metálicos, e incluso madera de grandes escuadrias, es relativamente nuevo incluso en paises con larga tradición de construcción con madera y, por supuesto, en España.

El hecho de que la producción de grandes escuadrias de madera se enfrente con una evidente limitación en su suministro, ha estimulado la investigación en este área al objeto de encontrar alternativas fiables a un precio competitivo. Se constata que la calidad de la madera disponible ha disminuido debido a la sustitución de la explotación de bosques maduros por otros más jóvenes, provenientes de plantación. Este factor en sí no es causa de una menor calidad, pero provoca el hecho de que la madera con unos crecimientos relativamente rápidos presente, en líneas generales, una menor densidad

Estos nuevos productos estructurales permiten la eliminación de nudos, grietas y características propias de la madera joven, mientras que la tasa de aprovechamiento de la materia prima es más alta. Es decir, se necesita menos volumen de fibra bruta para obtener las mismas o mayores producciones, y con superiores prestaciones que con madera maciza. El proceso de sustitución se está dando en EEUU a un ritmo muy vigoroso. Es previsible que también en Europa las aplicaciones en construcción de la madera maciza den paso a nuevos productos, tales como los laminados estructurales paralelos -Laminated Veneer Lumber (LVL) (Fig. 3)-, la madera reconstituida-Parallel Strand Lumber (PSL) (Fig. 4), Laminated Strand Lumber

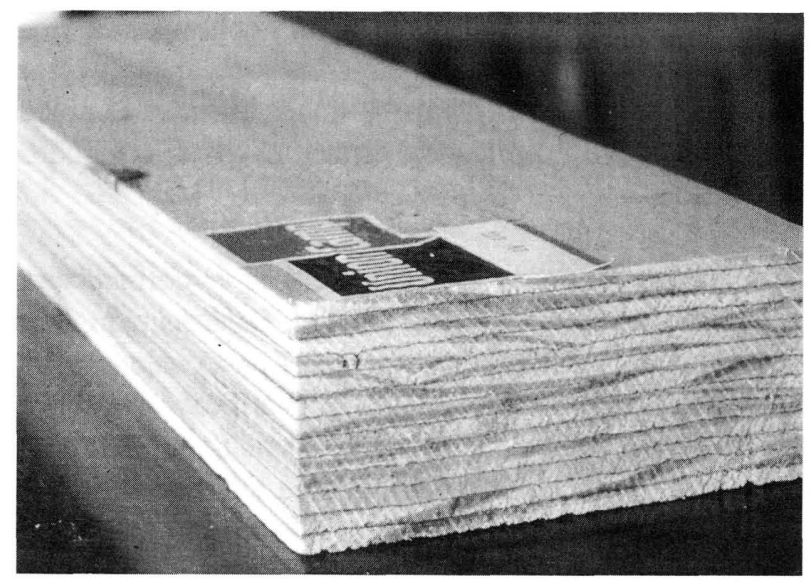

Fig. 3.- Pieza de laminado estructural paralelo. 
(LSL)-, la madera laminada encolada y las viguetas industrializadas de madera (I-Beams)'.

Para dar una idea de los avances conseguidos en este campo puede servir el cuadro $n^{\circ} 1$, en el que se incluye una comparación de las tensiones de cálculo de varios de los nuevos productos sustitutivos de la madera maciza.

Los productos industrializados estructurales encuentran sus principales aplicaciones en construcción residencial y comercial. A menudo se utilizan como componentes de un sistema integrado, loque mejorael rendimientodel conjunto. Por ejemplo, un forjado con estos materiales se puede componer de viguetas (I-Beams) arriostradas en cabeza o colgando de piezas perimetrales de madera reconstituida

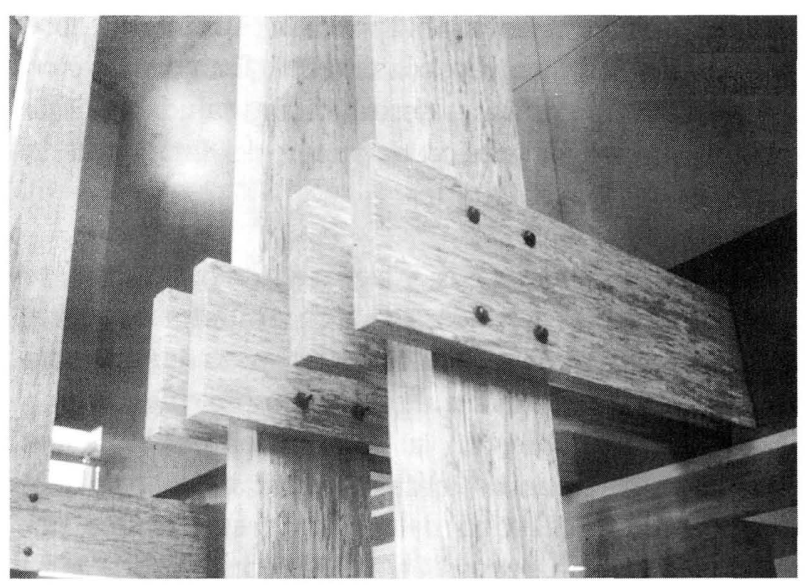

Fig. 4.- Unión de pilar y viga con PSL. Edificio FORINTEK (Canadá).

CUADRO $n^{0} 1$

\begin{tabular}{||c|c|c|||}
\hline \multicolumn{1}{||c||}{ COMPARACIÓN DE TENSIONES DE CÁLCULO DE MADERA DE CONSTRUCCIÓN Y PRODUCTOS } \\
SUSTITUTIVOS
\end{tabular}

(Laminated Strand Lumber), con apoyos intermedios sobre vigas de madera laminada, siendo el conjunto cubierto con tablero contrachapado, machihembrado, encoladoy clavado a las viguetas, o bien OSB, formando la base del forjado. Estos materiales, de los que se conocen con exactitud sus prestaciones, mejoran las características del elemento equivalente en madera $\mathrm{y}$, en muchos casos, de otros sistemas de construcción convencional de uso similar. En Norteamérica, es frecuente el empleo de paneles prefabricados de cubierta -stressed skin panels- en combinación con madera laminada, para edificios comerciales e industriales de cubierta plana. A este respecto, son ya clásicos los edificios religiosos y deportivos de cubiertas plegadas.

Otro producto empleado crecientemente en construcción es el panel sandwich, similar, en su concepto, al metálico. La diferencia radica en la composición de las láminas exteriores, normalmente de tablero OSB. Este tipo de construcción ha sido utilizado en España con unos resultados satisfactorios en la construcción de viviendas unifamiliares.

\section{La pared transpirante (Breathing Wall)}

El problema de la condensación de vapor en el interior de los paramentos sometidos a una diferencia de temperatura significativa a ambos lados siempre ha merecido una atención especial, por el potencial peligro que este fenómeno físico -la difusión de vapor de agua a través de medios porosos- plantea. En la hipótesis de que se dieran condensaciones de este vapor en el interior del paramento, se crearían las condiciones iniciales susceptibles de causar daños en la estructura a medio y largo plazo por la acción de los hongos o incluso insectos xilófagos. Por ello se procura que la disipación del vapor de agua en su migración a través de la pared sea lo más rápida posible. De aquí arranca el concepto de pared transpirante (Breathing Wall). En general, se entiende por ello un diseño de pared o cubierta carente de barrera de vapor y una disposición de las diversas capas de materiales que la componen de manera que la que presenta una permeabilidad al vapor de agua más baja se sitúa la primera en el lado cálido y, en orden de permeabilidad creciente, los demás materiales hacia el exterior del elemento. Como regla práctica se

1 La terminología castellama utilizada en este artículo no está reconocida ni consagrada por el uso, por lo que se mantienen, asimismo, sus siglas en inglés. 
establece que la resistencia al vapor de la capa interna debe ser del orden de cinco veces la externa. Esta disposición debe permitir una difusión desinhibida y saludable de la naturalmente mayor presión de vapor, del interior de la construcción hacia el exterior.

En algunos países europeos, y siguiendo esta filosofía, se utilizan los paneles de madera en la cara interior del muro, por considerar que los paneles tienen una muy baja permeabilidad al vapor. En algún sistema de construcción se propugna, simplemente, su eliminación por la misma razón. Investigaciones recientes apuntan al hecho de que, en el movimiento del vapor de agua a través de muros, tiene una importancia entre 100 y 1.000 veces mayor, medida en cantidad de agua, la falta de estanqueidad. Es decir, es mucho mayor el volumen de vapor de agua transportado por convección que por difusión. De aquí se deduce la importancia que para una adecuada conservación de las estructuras de madera en vivienda tiene la estanqueidad de los elementos perimetrales, y la necesidad de una esmerada ejecución para prevenir fugas de aire caliente al exterior, de forma no controlada, a través de paredes y cubiertas.

En cuanto a los materiales utilizados, este principio se mantiene instalando una barrera de vapor en el lado caliente, inmediatamente detrás del acabado de pared y cosido a la estructura de madera. En los espacios entre montantes se introducen productos aislantes. Al exterior, se adosa contra la estructura de madera un tablero estructural. La disposición de este elemento es lo que se ha discutido junto con la presencia de la barrera de vapor. Según ensayos de laboratorio recientes, se ha comprobado que el tablero ofrece una permeabilidad suficiente para la ventilación de la cavidad. En definitiva, tanto si se coloca barrera de vapor como si no, la presencia del tablero de forro adosado a la estructura de madera por su parte externa no impide la difusión del vapor de agua generado en el interior.

\section{Cimentaciones de madera}

El uso de pilotes de madera está ampliamente documentado desde la antigüedad. Se han desenterrado piezas utilizadas por los pueblos lacustres en Suiza de unos 4.000 años. Se sabe que los fenicios empezaron a tratar pilotes por medio del fuego y la brea y que los romanos desarrollaron toda una tecnología al respecto, según describe Vitrubio hacia el año 58 D.C. El Campanile de San Marcos en Venecia se asienta sobre pilotes de más de 1.000 años. Asimismo, se encuentran estructuras en buenas condiciones sobre pilotes sin ningún tratamiento que superan el siglo, por todo Estados Unidos. En todos estos casos, obviamente, no se dan las condiciones para el desarrollo de los hongos de pudrición: aire, humedady temperaturas adecuadas. Cuando la madera se sitúa en el suelo por debajo del nivel freático no se dan las condiciones para su degradación por hongos de pudrición. Hoy en día, se considera una vida útil de unos 100 años para un pilote de madera tratada

(c) Consejo Superior de Investigaciones Científicas

Licencia Creative Commons 3.0 España (by-nc)

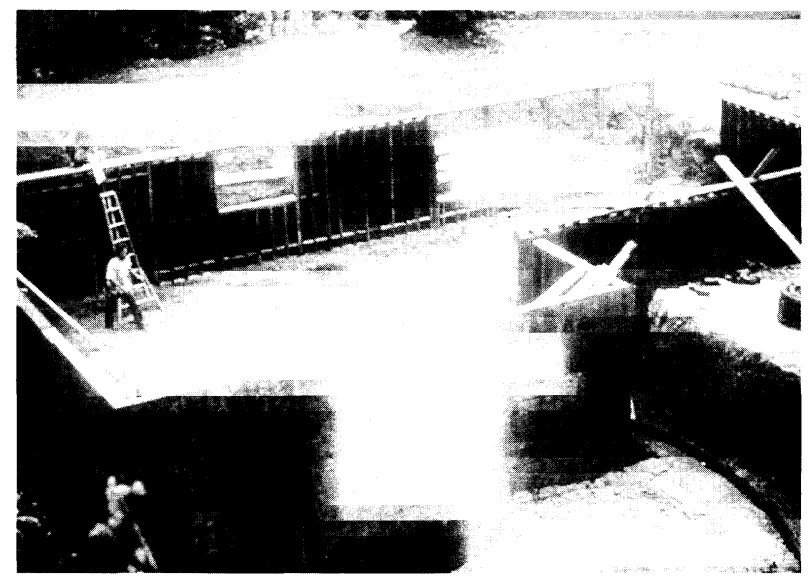

Fig. 5.- Muro de cimentación para sótano, en madera tratada.

El muro de cimentación de madera tratada (Fig. 5) es una aplicación relativamente nueva, siguiendo las posibilidades abiertas por los tratamientos químicos mejorados.

$\mathrm{Su}$ justificación práctica se da en lugares en que, por las especiales condiciones climáticas o de suelo, las cimentaciones convencionales de obra son dificiles de ejecutar o su coste se eleva extraordinariamente. Ejemplos de ambos se dan en zonas muy frías en las que el hormigonado se puede ver perjudicado por las bajas temperaturas, obien suelos permanentemente encharcados o de nivel freático alto.

En estas condiciones se puede plantear una estructura de madera que sustituya a la cimentación clásica. Las ventajas de orden práctico en cuanto a la ejecución de un cimiento de madera son evidentes:

-rapidez de ejecución

-utilización de herramienta manual de carpintería -no dependencia de las condiciones climáticas y edáficas del lugar

Desde el punto de vista de su comportamiento estructural, se pueden destacar también algunos inconvenientes:

-carece de la masa suficiente para evitar el vuelco del conjunto de la estructura en condiciones extremas de viento

-presenta limitaciones en cuanto a su capacidad de carga (empujes del terreno)

-se precisa un buen contacto con el suelo portante

Por último, se debe prestar mucha atención al material de relleno y sistema de drenaje del suelo y a la protección del conjunto con membranas impermeables.

El empleo de maderas tratadas resulta obligado en esta aplicación. Lo más frecuente es el tratamiento en autoclave, con sales en medio acuoso. Éstos tienen la ventaja, frente 
a los tratamientos con creosotas y otros productos orgánicos, de que no desprenden gases ni olores y quedan permanentemente fijados a la madera. Los productos de tratamiento son combinaciones de sales inorgánicas (cobre, arsénico, boro). Estos compuestos quedan fijados a las fibras de la madera y no son lixiviados por el contacto permanente o periódico con agua. Atención especial se debe dedicar a las uniones madera-tablero en contacto con el terreno. Ensayos acelerados de laboratorio concluyen que, excepto en el caso del acero inoxidable, otros medios metálicos de unión pueden deteriorarse en contacto con productos tratados, en las condiciones de humedad habituales del terreno. El tablero contrachapado y la madera deben trabajar solidariamente ante las solicitaciones debidas a posibles movimientos del terreno y su empuje, vientos y otras cargas circunstanciales, además de restringir el pandeo de los montantes que soportan la carga del edificio, por lo que las uniones metálicas deben ser inalterables a la acción química.

Básicamente, se consideran dos tipos de diseños para la cimentación, según se trate de pared simple - el arranque del muro está parcialmente enterrado- o reforzada con jabalcones, trabajando a compresión en el caso de un forjado de tipo sanitario. Si se trata de un sótano, es necesario prevenir el empuje del terreno en la base del muro. Para ello se apea directamente sobre el suelo y contra el paño enfrentado la línea de muro. En caso que la luz sea excesiva, se aprovecha la posible existencia de muros de carga para acortar luces. Si ello no es posible, habría que arriostrar el conjunto por los procedimientos habituales.

La dimensión de la madera necesaria para un muro de arranque suele ser de $38 \times 140 \mathrm{~cm}$, en edificios de dos alturas, y de $38 \times 89 \mathrm{~cm}$, para una altura sobre rasante. El espaciamiento entre montantes suele ser cada 30 ó $40 \mathrm{~cm}$. Los gruesos de tableros contrachapados más habituales son de 16,19 y $22 \mathrm{~mm}$, para los mismos espaciamientos entre montantes. Con ello se obtienen resistencias al empuje del terreno del orden de $1.500 \mathrm{~kg} / \mathrm{cm}^{2}$.

\section{Madera laminada encolada}

Entre las primeras experiencias conocidas sobre la utilización de la madera laminada encolada en Europa, está la construcción de un Auditorio en Basilea (Suiza), en 1893. La estructura se resuelve a base de arcos, utilizando probablemente caseína como resina de encolado. Más adelante, la madera laminada encontró también aplicación en otros campos tecnológicamente avanzados como la aviación y reafirmó sus posibilidades en el sector de la construcción. Con el desarrollo de colas estables, en la década de los cincuenta, se hizo posible su utilización en puentes, construcciones marinas y ambientes agresivos.

En cuanto a la forma y tamaño de las piezas, no existen otras limitaciones que las dictadas por el transporte, manipulación y puesta en obra. Las láminas en la pie(c) Consejo Superior de Investigaciones Científicas

Licencia Creative Commons 3.0 España (by-nc) za terminada se pueden disponer verticalmente, aunque lo más frecuente es hacerlo de forma horizontal. Con arcos, se han llegado a salvar luces por encima de los 100 metros. En piezas rectas, no es raro sobrepasar los 30 metros.

Son numerosas las ventajas de este producto, entre las que se puede citar:

-facilidad de fabricación de elementos de gran tamaño. partiendo de madera fácilmente disponible.

-minimización de grietas y otros defectos asociados a la pérdida de humedad de la madera, en piezas de gran sección.

-mayoración de las resistencias de cálculo de la madera y posibilidad de utilización de maderas de diferente calidad, en función de la disposición de las láminas en la pieza acabada.

Este material encuentra sus mejores aplicaciones en piezas sometidas a flexión, pilares, arcos, barras de cerchas y estructuras de cubierta. Debido al proceso de homogeneización resultante del empleo de madera clasificada para las láminas y a la posibilidad de saneado como paso previo a su fabricación, el producto terminado admite unas tensiones de cálculo superiores a las de la madera de partida.

El comportamiento del elemento terminado se basa, principalmente, en la naturaleza y calidad del encolado Paragarantizar este aspecto crucial, todas las variables que puedan influir sobre éste durante el proceso de fabricación habrán de ser rigurosamente controladas, incluyendo los parámetros ambientales (temperatura y humedad)

La madera laminada encolada ya es conocida y estimada en la construcción española, sobre todo en el ámbito de la obra pública. La existencia de una producción nacional, junto con la presencia en el mercado de otros fabricantes foráneos, está sin duda influyendo positivamente en la popularización de su uso.

\section{Puentes de madera}

Los avances tecnológicos asociados al empleo de la madera como material estructural y el desarrollo de nuevos sistemas de puentes a base de componentes de madera, están resituándola como un competitivo material de construcción para puentes (Fig. 6). Estos nuevos conceptos incluyen el empleo de elementos laminadosy tensionados de madera, madera laminada encolada y composites de madera en configuración de secciones en $\mathrm{T}$.

En Norteamérica, la administración federal de carreteras estima que la mitad, aproximadamente, de las más de 500.000 estructuras de puentes existentes en la red viaria son funcionalmente obsoletos o padecen defectos 


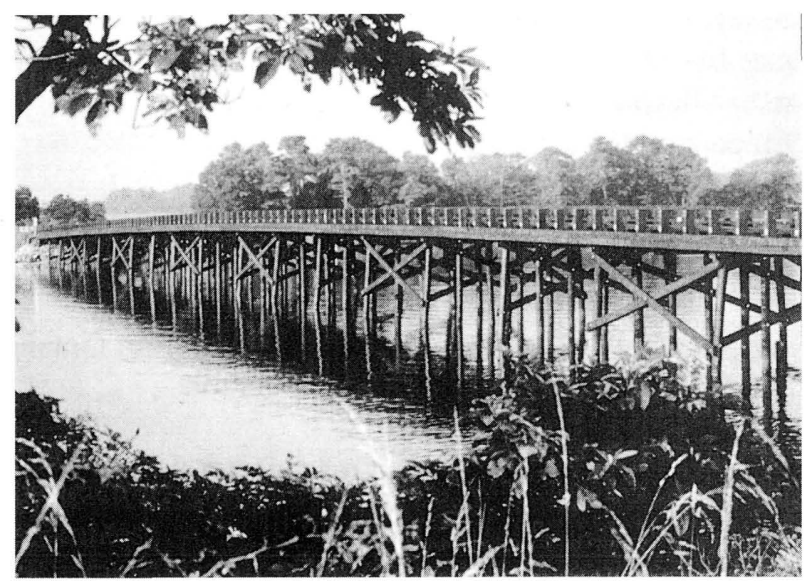

Fig. 6.- Puente WYE Narrows, para tráfico rodado. QueenAnne's County, Maryland (EEUU).

estructurales, requiriendo por tanto extensos trabajos de reparación o sustitución para adaptarse a la normativa en vigor. Muchos de ellos sobrepasan los 15 metros de luz y están situados en carreteras secundarias. Al depender su conservación de las administraciones locales, su mantenimiento suele ser deficiente. Estas circunstancias se combinan de forma que los sistemas modernos de construcción de puentes de madera se convierten en una buena alternativa

La realidad es que la mayoría de los expertos consideran que la madera no es un buen material para este uso, si se compara con el hormigón o con el acero. Se percibe la madera como un material obsoleto, apto únicamente para la construcción de viviendas. Existe una abundante experiencia de estructuras de madera de todo tipo dañadas por el paso del tiempo. En la mayor parte de los casos referentes a puentes de madera se producen problemas asociados a un tratamiento de protección inadecuado o a diseños defectuosos en sus detalles. El diseño habitual de los puentes deteriorados utiliza un tablero a base de laminados de madera con clavos. Los daños consisten en el aflojado de las uniones entre laminados, la deformación excesiva del tablero y pudriciones. Con la aparición de la madera laminada encolada se produjo un importante avance de la tecnología constructiva, mejorando con ello el tablero del puente. Algunos de los primeros puentes construidos en Norteamérica con esta técnica, apenas finalizada la II Guerra Mundial, se acercan a los 50 años en servicio.

El diseño típico de puentes con madera laminada para tráfico vehicular consistía en varias piezas longitudinales de madera laminada salvando la luz y un tablero transversal de piezas de madera laminado a clavo. También se utilizaron otras soluciones mixtas, con hormigón o de hormigón exclusivamente, como tablero. Ninguna de estas propuestas resuelve el problema de entrada de agua a través del (c) Consejo Superior de Investigaciones Científicas tablero sobre las piezas maestras. El tablero laminado a clavo se consideró una solución aceptable para luces cortas, típico de los puentes de madera maciza en que el tablero se apoyaba sobre vigas maestras muy próximas. La posibilidad de mayores vanos con la madera laminada elevó las exigencias sobre el tablero. Ello condujo a la definición de un tablero de puente de madera laminada. La disposición de las láminas en el tablero es ortogonal a la cara del tablero, en paralelo a la dirección de la carga. Además de conseguir una mayor rigidez del elemento, se considera que proporciona una mejor protección ante la humedad al vigamen del puente. El ancho óptimo de las piezas del tablero se ha definido en 1,22 metros. Para asegurar la continuidad entre las piezas fue necesario estudiar mecanismos de unión que asegurasen una óptima transferencia de cortantes y momentos en los bordes de las placas.

Otra alternativa para puentes de hasta 10 metros consiste en laminar longitudinalmente las placas en paralelo al vano. Este sistema elimina la necesidad de apoyo de placas y se emplea para situaciones en que hay limitaciones de altura del tablero y se desea un perfil plano para la estructura.

\section{Vigas en I}

El concepto de viga en I a partir de madera y derivados está formulado, al menos, desde los años 50. Entonces se conocían como vigas-cajón, de las que ésta es un caso singular. Su introducción en el mercado norteamericano se ha producido por medio de una empresa que ha fijado el estándar para el resto de fabricantes.

Este elemento se fabrica a partir de dos componentes: material para las alas y para el alma. El primero consiste en madera empalmada por testa y clasificada estructuralmente con máquina -Machine Stress Rated (MSR)-, o bien con madera reconstituida estructural Structural Composite Lumber (SCL)- como en los chapados laminados en paralelo -Laminated Veneer Lumber (LVL) (Fig. 7)-, o bien la madera reconstituida en paralelo -Parallel Strand Lumber (PSL)-. Los últimos son preferidos sobre la madera empalmada debido a su mayor homogeneidad en la sección, carencia de juntas y propiedades mecánicas constantes. El material para el alma suele ser tablero contrachapado calidad construcción de $9,5 \mathrm{~mm}$, o tablero de viruta orientada OSB. El alma se inserta en una canal practicada en la cara ancha de las alas. Los tableros de las almas se encolan a tope entre sí para dar continuidad a ésta y también entre alas y almas, todo ello con colas estables a la humedad-fenólicas-. Las dimensiones de la viga se ajustan según la aplicación. Los cantos más corrientes varían entre, aproximadamente, 15 y $76 \mathrm{~cm}$. Debido a su fabricación en continuo se puede obtener en cualquier longitud aunque, por razones de manipulación, el máximo se sitúa en unos 24 metros. Su relativo bajo peso -entre 3 y $18 \mathrm{~kg}$ por metro lineal-, facilidad de uso y 


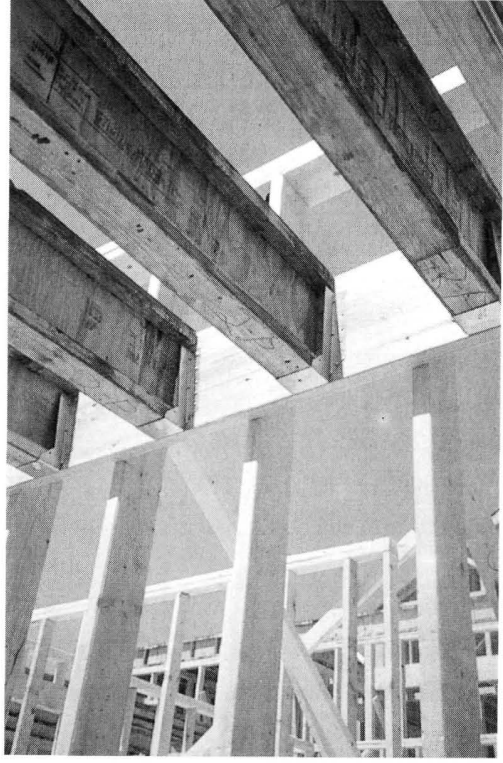

Fig. 7.- Detalle del apoyo de viguetas en I. Viviendas adosadas en Villanueva de la Cañada (Madrid).

flexibilidad de diseño lo convierten en un producto muy utilizado. Como nota adicional se puede mencionar la posibilidad de fabricación con canto de altura variable (Fig. 8).

Las viguetas que se utilizan para forjados no incorporan una contraflecha de fábrica por las relativamente cortas luces en que se emplean. Sin embargo, cuando se utilizan en tejados debe especificarse, particularmente si se trata de cubiertas planas. En este caso se recomienda una contraflecha mínima equivalente a la deformación por peso propio más la mitad de las sobrecargas. Los fabricantes proporcionan igualmente información sobre dimensión mínima de apoyos, necesidad de refuerzos en el alma para evitar su pandeo y transferir cargas puntuales, y

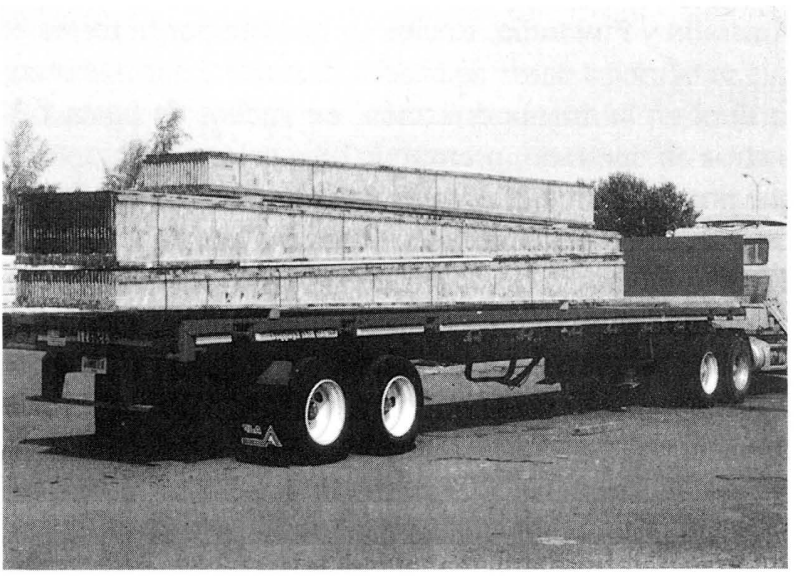

Fig. 8.- Viguetas de canto variable.

apertura de agujeros en la misma, su posición respecto a los apoyos, forma y tamaño.

Este producto está reconocido en los códigos de construcción norteamericanos sobre labase de documentos equivalentes a lo que los DIT (Documentos de Idoneidad Técnica) representan en España. A causa de que en su fabricación se utilizan diferentes materiales, y para permitir su intercambio entre viguetas de procedencia distinta, está próxima a aparecer una norma de aplicación uniforme reconocida por los principales códigos de construcción norteamericanos.

\section{Multilaminados paralelos -Laminated Veneer Lumber (LVL)-}

Este producto está referenciado en la literatura desde los años 40 , aunque su producción industrial no comenzó hasta los 70 (cuadro $^{\circ} 2$ ). En la actualidad se produce en varios países además de EEUU, como Canadá, Japón,

CUADRO $\mathrm{N}^{\circ} 2$

\begin{tabular}{|c|c|}
\hline \multicolumn{2}{|c|}{$\begin{array}{l}\text { PROPIEDADES DE CÁLCULO ADMISIBLES TÍPICAS DE LVL } \\
\text { (CONTENIDO DE HUMEDAD MENOR 15\%) }\end{array}$} \\
\hline CARACTERÍSTICA & RANGO DE CÁLCULO ADMISIBLE (MPa) \\
\hline $\begin{array}{l}\text { Flexión } F_{b} \\
\text { Tracción } F_{t}\end{array}$ & $\begin{array}{l}13,4-21,3 \\
9,6-17,9\end{array}$ \\
\hline $\begin{array}{l}\text { Compresión } \\
\text { paralela a la fibra } F_{c} \\
\text { perpendicular } F_{c}\end{array}$ & $\begin{array}{c}13,4-19,6 \\
2,4-5,3\end{array}$ \\
\hline $\begin{array}{l}\text { Cortante } \\
\text { rasante } \\
\text { perpendicular }\end{array}$ & $\begin{array}{l}1,1-1,7 \\
1,0-1,9\end{array}$ \\
\hline Módulo de Elasticidad (MOE) & $12.400-15.100$ \\
\hline
\end{tabular}


Australia y Finlandia. Recibe su nombre por la forma en que se fabrica a partir de chapas de madera encoladas con la fibra en la misma dirección, en anchos de hasta 1,22 metros en continuo, prensando bajo calor el conjunto, lo que proporciona una densificación al material resultante. De aquí se cortan las piezas en dimensiones estandarizadas. Es un producto adecuado para aplicaciones muy exigentes, ya que la multiplicidad de chapas ayuda a aleatorizar la presen- cia de los defectos característicos en la madera. El resultado es un producto de mejores prestaciones que la madera, reduciendo la dispersión de los valores de su resistencia desde un $30 \%$ en la madera aserrada, hasta un $10 \%$ en el LVL, lo que lo hace muy apto para vigas y componentes de cerchas y viguetas.

\section{La cercha de madera}

Se han usado durante cientos de años, aunque el comienzo de la producción de cerchas industrializadas en masa, ligeras, e integrando en muchas ocasiones elementos metálicos, ha cambiado totalmente la filosofía de diseño. Anteriormente al desarrollo de los sistemas de cerchas ligeras, era considerada como un elemento complejo de costosa fabricación a medida. Se puede entender que cada cercha trabaja de forma independiente respecto de las otras que forman el conjunto de la estructura, sin que constituyan un sistema propiamente dicho, debido a su relativamente gran espaciamiento

La introducción de los conectores o placas metálicas dentadas, junto con la posibilidad de empalmes de testa en piezas de madera para pares, ha conducido a una nueva filosofía en los sistemas de cerchas. Estos avances permiten la fabricación y uso de elementos muy ligeros y económicos en largas series. La disposición repetida, a muy corta distancia, permite sustituir a las antiguas cerchas de gran sección muy espaciadas. De esta forma, las cerchas se encuentran normalmente a distancias entre 30 y $120 \mathrm{~cm}$, clavando el tablero de cubierta directamentea los pares. Los nudos se resuelven en la práctica clavando dos placas a ambos lados, normalmente con prensa (Fig. 9). El tamaño de la placa debe cubrir todas las piezas que llegan al nudo, con un área efectiva de clavado en cada una tal que permita la transmisión efectiva de la cargas entre piezas, sin rebasar un valor dado por unidad de superficie en la placa dentada. Existen numerosos tipos de placas de unión con caracteristicas especificas. Los distintos fabricantes proporcionan la información necesaria para el cálculo y fabricación. Hay también otros formatos de cerchas mixtas, a base de pares de madera y celosía metálica. Normalmente, son sistemas patentados

El diseño de un sistema de cerchas debe tener en cuenta las diversas solicitaciones posibles comunes a otro tipo de materiales. La forma en que éstas se aplican o son soportadas pueden ser específicas de la madera. Las cargas a que se someten son las cargas propias y las sobrecargas -cargas

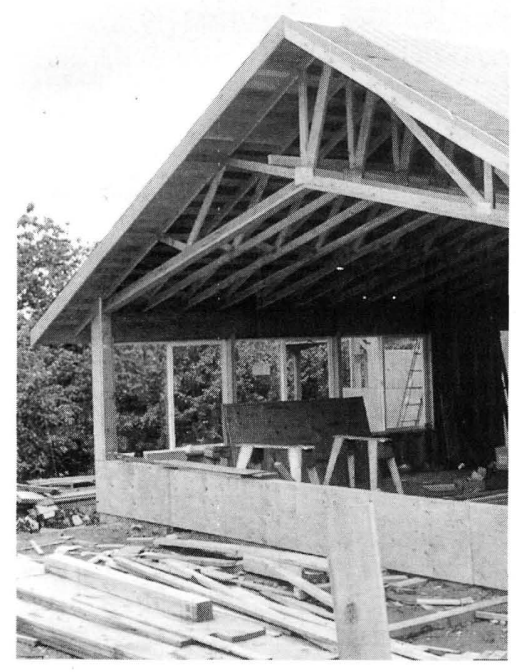

Fig. 9.- Cerchas en tijera, en edificio comercial. Washington (EEUU).

vivas, nieve, viento, sismo, tensiones en el montaje-. En las cubiertas planas se debe prestar atención a la formación de charcos de lluvia por la posibilidad de colapso de la cubierta. Cuando la deformación se inicia por esta causa puede continuar a mayor velocidad que la resistencia de la cercha, hasta que se restablece el equilibrio o bien se produce el fallo. Finalmente se deben prever las cargas concentradas debidas a la instalación de equipos pesados. Otra consideración necesaria en estos sistemas se refiere a la duración de la carga: desde una duración de 10 años para cargas permanentes, hasta de 10 minutos, con una mayoración de esfuerzos admisibles de hasta un $60 \%$ en el caso de viento o sismo

Como consecuencia del comportamiento diferenciado de la madera bajo carga permanente es necesario prever la deformación a corto y largo plazo. Los límites son diferentes para tejados y forjados. Para lo primeros, el criterio es el de procurar la evacuación del agua con una consideración que prevea posibles daños en el acabado de los cielorrasos sujetos de forma directa a las cerchas. Se establece entre 1/360 y 1/240 de la luz, según el tipo de material de acabado. En el caso de forjados, se suelen requerir límites más estrictos para la deformación. Para sobrecargas de uso no permanentes, se sitúa en 1/360 en forjados tradicionales aunque, con el aumento de las luces por el empleo de elementos más esbeltos, se tiende a 1/480 para uso residencial.

La percepción sensorial al comportamiento de los forjados de madera requiere una atención muy particular. Aunque es un tema complejo, se evidencia la necesidad de asegurar una elevada rigidez que elimine las objeciones al movimiento o vibración en el forjado. En este sentido, se recomienda tomar las siguientes medidas: 
-asegurar una perfecta instalación de las piezas -utilizar unos límites a la flecha más rigurosos que los obtenidos por cálculo

-tener en cuenta el efecto combinado entre el tablero del forjado y el vigamen (particularmente si se instala encolado y clavado)

-utilizar tableros estructurales más gruesos de lo indicado por cálculo

-arriostrar la parte inferior de las vigas

- cargar los forjados adecuadamente

-planificar discontinuidades estructurales entre divisiones de propiedad

-utilizar materiales elásticos como recubrimientos de suelos.

En general, la sensibilidad humana ante el comportamiento del forjado crece con el costo y sofisticación del edificio. Igualmente, una vez que una persona se sensibiliza ante el pobre comportamiento de un forjado resulta difícil contrarrestarlo con medidas correctoras posteriores. Por ello, es muy importante la reacción inicial del usuario al comportamiento de un suelo.

\section{Situación de la normativa de construcción con madera en España}

El panorama normativo europeo sobre la madera en la construcción está experimentando una rápida actualización, en línea con los numerosos requerimientos normativos que el mercadoúnico exige. En esteámbito, se está desarrollando un cuerpo común de normativa de cálculo para estructuras que abarca todos los materiales utilizados en construcción, incluyendo la madera. El volumen dedicado a la madera es el Eurocódigo 5, siendo ésta una norma de cálculo de obligada publicación por los países miembros del Comité Europeo de Normalización (CEN). El Comité Técnico CEN 250, Eurocódigos, cuyo correspondiente en España es el CTN 140, del mismo nombre, aprobó en 1993 la parte 1.1 -Proyecto de Estructuras de Madera.Reglas Generales-, que se encuentra pendiente de publicación en español; la parte 1.2 -Cálculo del Comportamiento frente al Fuego-, fue aprobadaen 1994 y no hay versión en español todavía. Finalmente, la parte 2 -Puentes- está en borrador.

Las normas de referencia del Eurocódigo 5 se elaboran en diferentes Comités Técnicos del CEN. Así el CENTC 124, Estructuras de Madera, se encarga de redactar normas sobre los siguientes aspectos:
Grupo 1. Métodos de ensayo para:
-Madera Aserrada
-Madera Laminada
-Tableros de madera
-Paneles Compuestos
-Elementos de unión

Grupo 2. Madera Maciza. Normas sobre

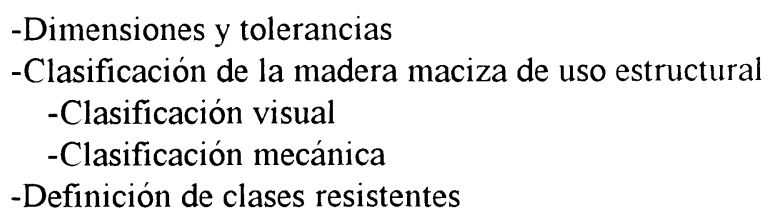

Grupo 3. Madera laminada-encolada. Normas de:

-Fabricación
-Ensayo de encolados

Grupo 4. Uniones:

-Clasificación de uniones

-Capacidad de los medios de unión

El CEN TC 112 desarrolla las normas que afectan a los tableros de madera (de partículas, de fibras, contrachapados, etc). y se organiza, a su vez, en 6 grupos de trabajo. Existen otros comités técnicos que realizan aportaciones como el 38 , sobre tratamientos de madera o el 175 , sobre madera aserrada de uso no estructural.

España participa en este trabajo de normalización a través del Comité Técnico de Normalización CTN 56, de AENOR.

Aunque se partía de una base normativa casi inexistente en lo que se refierea las aplicaciones estructurales de la madera se prevé que, en un plazo de entre 4 y 8 años, se contará con toda la normativa básica y de referencia para implantar con garantía estos sistemas de construcción.

Asimismo, ya hay preparado un borrador de una futura NBE-Estructuras de Madera, basado en el Eurocódigo 5 y en las normas aprobadas por el CEN TC 124, que una vez examinado y aprobado pasará a ser de obligado cumplimiento para la construcción con madera en España.

\section{BIBLIOGRAFÍA}

(1) R. ARGÜELLES et al.: "Construcción en Madera”. COAM, 1988

(2) R. ARGÜELLES y F. ARRIAGA: "Curso de Diseño y Cálculo de estructuras de Madera”. COAM, 1988.

(3) F. ARRIAGA et al: "Guía de la Madera en la Construcción". AITIM, 1994. 
(4) FAHERTY y WILLIAMSON: “Wood Engineering and Construction Handbook”. McGraw-Hill, 1995.

(5) K. IRLE y C. LOXTON: "The Manufacture and Use of Panel Products in the L. . Institute of Wood Science. 1996 Annual Conference.

(6) M. MILNER y G. PITTS: "Misconceptions about Breathing Walls". The Arcinitects journal, March 1995.

(7) O'HALLORAN: “Changing Nature of Wood Products”. Construction and Building Materials, vol. 6, № 3, 1992.

(8) J.E. PERAZA et al.: "Casas de Madera". AITIM, 1994.

(9) M. RITTER et al.: "Performance of Stress-Laminated Bridges". Wood Design Focus, vol. 1, No 3, 1990.

(10) T. WILliAMSON: “Engineered Wood Group Pushes Standard I-Joist Code”. Journal of Light Construction, May 1995.

(11) T. WILLIAMSON: "Glued Laminated Timber for Bridge Construction”. Wood Design Focus, vol. 1, № 3, 1990.

(12) Breathing Wall: Design Concepts. Excel Industries Ltd. Commercial brochure, 1994.

(13) Plywood Design Specification. APA The Engineered Wood Association, 1986.

(14) Vapor Permeance Tests of APA Trademarked Plywood, APA Internal Report, 1996.

\section{Publicación del Instituto Eduardo Torroja - CSIC}

\section{Número monográfico de INFORMES}

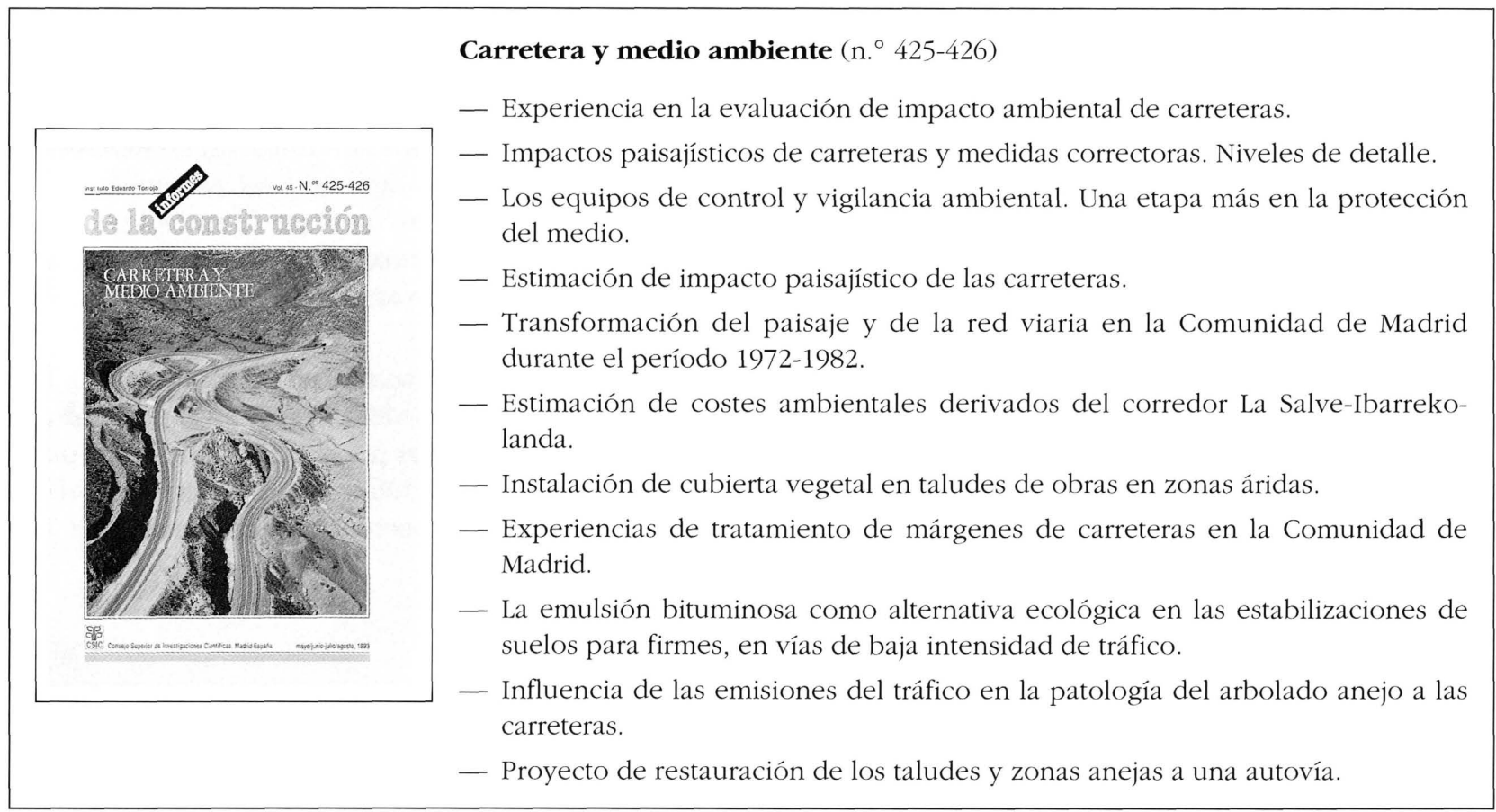

